\title{
ORAL SQUAMOUS CELL CARCINOMA WITH PSEUDO-GLANDULAR DIFFERENTIATION
}

S.W. Chan, F.K. Hartanto, L. Auzair, N.F. Mohd Tahir,

N. Harun, L.O. Aung, C.H. Siar. Oral Squamous Cell Carcinoma with pseudo-glandular differentiation. Annal Dent Univ Malaya 2013; 20(2): 29-33

\begin{abstract}
Conventional oral squamous cell carcinomas are readily recognized histopathologically but the presence of additional atypical features may be challenging from a diagnostic point of view. We present a case of a welldifferentiated oral squamous cell carcinoma with pseudoglandulardifferentiation and discuss thepossibledifferential diagnoses on a histopathological basis. Accurate diagnosis is imperative for timely and appropriate intervention and denotes distinctive prognostic implications. The presence of perivascular and perineural infiltrations as observed in this case would indicate the need for further post-operative therapeutic decision-making aimed at controlling local spread as well as distant metastases.
\end{abstract}

Keywords: Oral squamous cell carcinoma, pseudoglandular, adenoid, gland-like, histopathology, diagnosis

\section{INTRODUCTION}

Squamous cell carcinoma (SCC) is the most common form of oral malignancy and majority present as welldifferentiated or conventional keratinizing squamous cancers. However, additional characteristic histological features and clinical behavior distinguish the rare variants or subtypes of SCC from the conventional SCC. These constitute in aggregate, approximately $10-15 \%$ of all SCCs in the upper aerodigestive tract (1). Histologic subtypes of oral SCC include spindle cell carcinoma (sarcomatoid), basaloid squamous cell carcinoma, adenosquamous carcinoma, adenoid squamous or acantholytic carcinoma, papillary or exophytic squamous cell carcinoma, verrucous carcinoma and carcinoma cuniculatum $(1,2,3)$. Besides academic interest, recognition of these subtypes is vital as each exhibits unique histomorphological appearance which raises several differential diagnostic considerations, and distinctive prognostic implications (1).

\section{CASE REPORT}

A 69 year old Chinese man presented to the Department of Oral and Maxillofacial Surgery (OMFS), Faculty of
Case Report

S.W. Chan, F.K. Hartanto, L. Auzair, N.F. Mohd
Tahir, N. Harun, L.O. Aung, C.H. Siar
Department Of Oro-Maxillofacial Surgical and
Medical Sciences, Faculty of Dentistry, University
of Malaya, Kuala Lumpur, Malaysia
Corresponding Author:
Dr. Chan SiewWui
Email: swuichan@gmail.com

Dentistry, University Malaya on the $23^{\text {rd }}$ of March 2011 with a complaint of a painless, progressively growing ulcer at the right lower jaw for the past 4 months. The patient was a non-smoker but suffered a myriad of medical problems including hypertension, benign prostatic hyperplasia, unrepaired right inguinal hernia, chronic constipation, chronic hyponatremia, major depression complicated by obsessive-compulsive disorder-like symptoms, suspected early dementia, treated glaucoma and cataract. He also had a history of repeated falls and sustained fractured fingers and clavicle.

Extraoral examination showed that the right submandibular lymph node was palpable, mobile, nontender and measured about $1 \mathrm{~cm}$ in diameter. A dental panoramic radiograph and a lower occlusal radiograph did not show any underlying bony involvement. Upon intraoral examination, there was an ulcerated swelling at the buccal sulcus in the region of teeth 45,46 and 47. It measured approximately $5 \mathrm{~cm} \times 5 \mathrm{~cm}$ and extended upwards towards the upper dentition and lower edentulous area. Tooth 47 was displaced lingually and had grade II mobility. The base of the swelling was indurated but the surface was friable. An incisional biopsy was taken from the ulcerated swelling and was submitted for histopathological examination. Subsequent microscopic evaluation revealed a well-differentiated SCC arising from the overlying dysplastic epithelium and randomly invading the underlying connective tissue. The tumour epithelium exhibited well-differentiated squamous cells, keratin pearl formation and distinct intercellular bridges. Mitoses were less than 2 per $10 \mathrm{HPF}$ (high power field). The invasive front was blunt, presenting in a Type 1 patternA mild chronic inflammatory cell infiltrate was present. Adjacent to the tumour was normal epithelium. Lymphovascular permeation was not evident. 

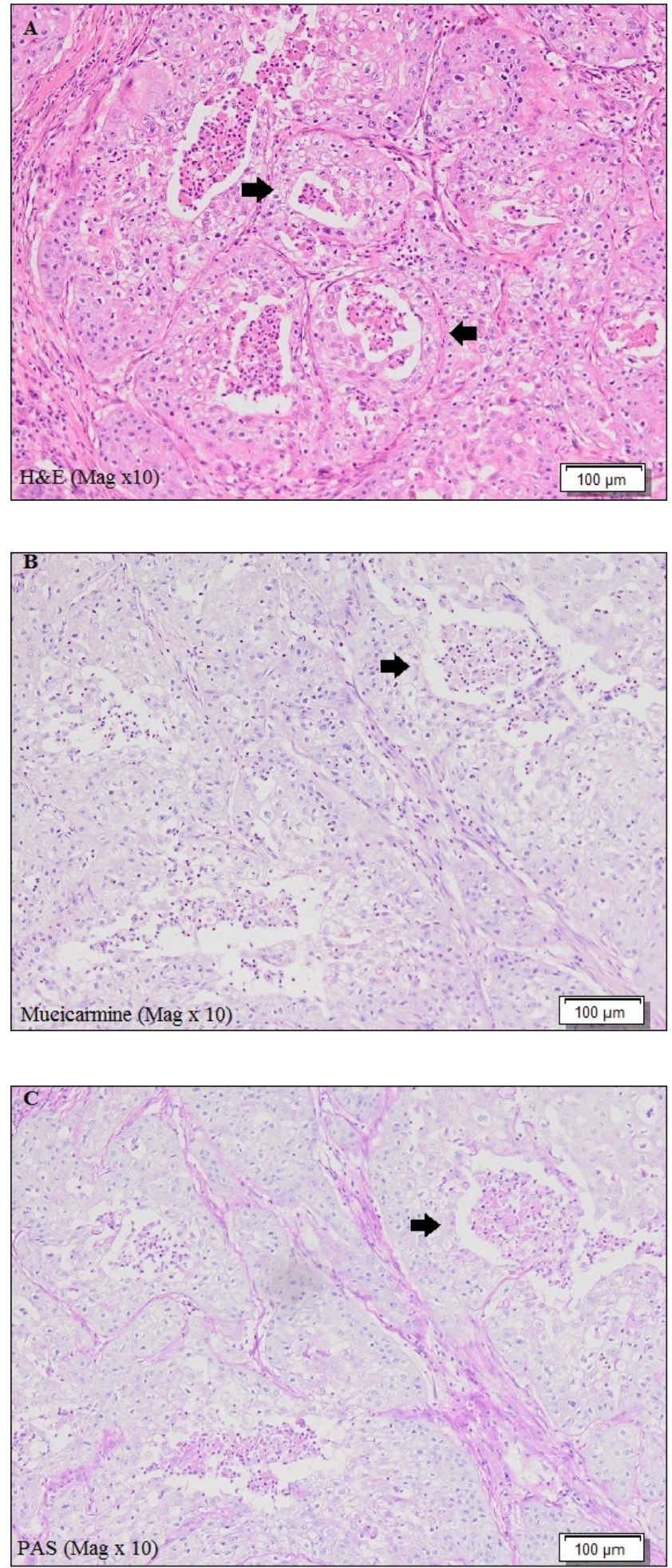

Figure 1: (A) Pseudoglandular appearance of neoplastic epithelium due to comedo necrosis. Pseudoglandular structures (arrows) stain negative for Mucicarmine (B) and PAS (C).
CT (computed tomography) scan found a $2.5 \times 2.3$ $\mathrm{x} 2.3 \mathrm{~cm}$ mass eroding the right mandible which was consistent with a carcinoma but there was no evidence of metastasis. A wide excision of the tumour with marginal mandibulectomy was done on the $11^{\text {th }}$ of April 2012 with post-operative radiotherapy to follow.

Histopathological examination of the surgical specimen returned a diagnosis consistent with the biopsy report. Sections of the main tumour showed a solid malignant epithelial neoplasm with an exophytic fungating component and an endophytic component that extended deep into the submucosal tissues. The neoplastic epithelium showed an admixture of squamous and pseudoglandular differentiation (Figure 1).

Keratin production was moderate to marked. Mitotic count was 3 per $10 \mathrm{HPF}$. Lymphovascular invasion was not evident. The tumour advancing front consisted of large tumour islands and smaller clusters randomly invading the submucosa. Tumour extended to involve the surgical margins. A diffuse chronic inflammatory cell infiltrate was present in the intervening stroma. Special stains did not show the presence of mucous-secreting cells within the tumour epithelium. There were tumour nests juxtaposed to the blood vessel wall and epineurium in some areas from the specimen labeled inferior alveolar nerve (Figure 2 ). Tumour involvement of the bone was evident with positive marrow spread but submandibular lymph nodes were free of tumour.

Patient was last seen in OMFS clinic two weeks post operatively.

\section{Perivascular invasion}

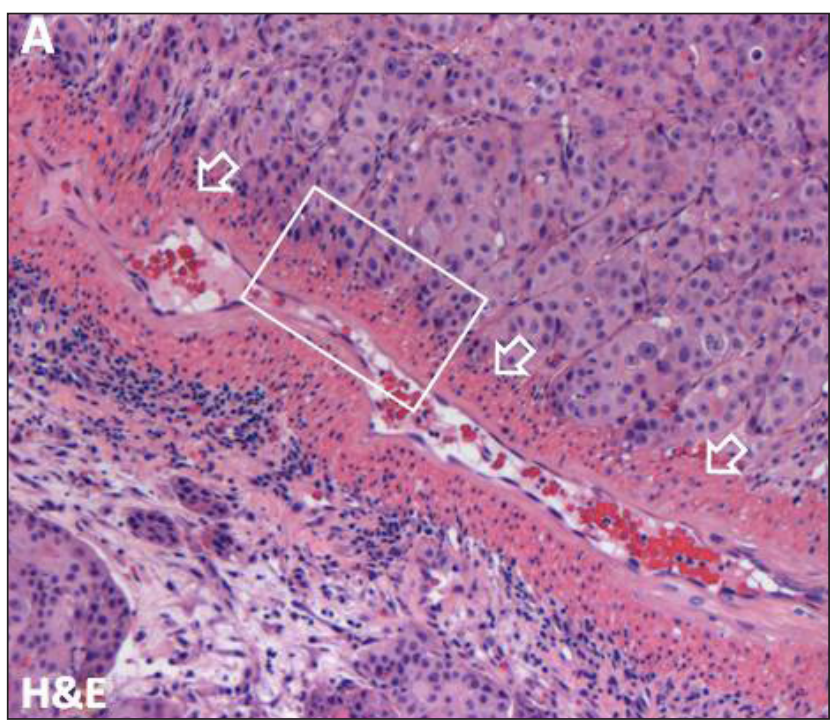

Figure 2: H\&E stains demonstrate prominent perivascular invasion by neoplastic epithelium [A] (Mag x 10), 


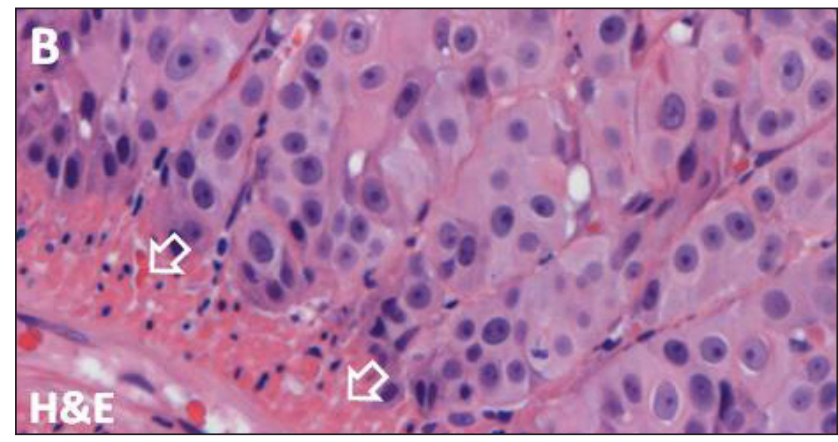

\section{Perineural invasion}
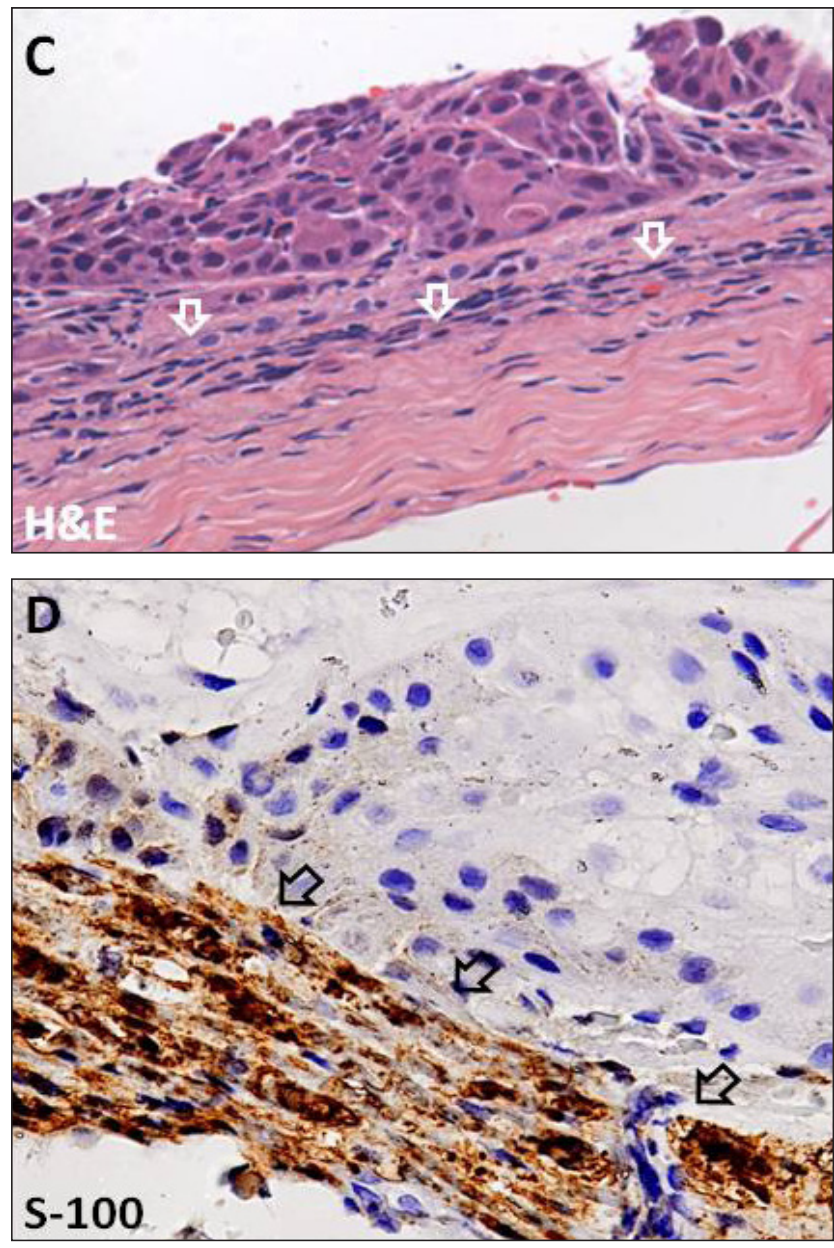

Figure 2 (contd): Details of perivascular invasion by neoplastic epithelium [B] (Mag x 40)]. (C) Tumour nests juxtaposed to the epineurium in an H\&E section [Mag $x 20$ ] (D) S-100 immunostaining highlights perineural involvement of the tumour nests [Mag $\times 40]$.

\section{DISCUSSION}

In the present case, the neoplastic epithelium showed an admixture of squamous and adenoid (gland-like) features prompting several differential diagnoses. Adenoid features could be present in several types of carcinoma, complicating the interpretation of tissue sections which may lead to misdiagnosis. Differentiation among these neoplasms is essential to ensure that appropriate clinical management is administered (4). Malignancies in consideration are the conventional SCC with or without ductal involvement, adenosquamous carcinoma, acantholytic SCC and basaloid SCC. Minor salivary gland neoplasms such as adenoid cystic carcinoma, mucoepidermoid carcinoma and adenocarcinoma with squamous metaplasia should be ruled out as well $(2,4,5)$.

Adenosquamous, basaloid and acantholytic variants of SCC are biphasic, with squamous-type carcinoma being a consistent feature, lending the appearance of conventional SCC in the superficial part $(1,2)$. Adenosquamous carcinoma of the head and neck was first defined by Gerughty et al. in 1968 but its existence as a distinct entity remained controversial formany years as some investigators regarded it a high grade mucoepidermoid carcinoma (6). However, separation between the two entities is merited in view of the highly malignant and aggressive nature of adenosquamous carcinoma with markedly worse prognosis compared to mucoepidermoid carcinoma, as well as distinct morphologic and clinical differences (7). The precise origin of this tumour is also subject to debate with opinions divided between a mucosal origin versus a salivary gland duct origin. Regardless, there is little doubt that an origin in surface mucosa, involving squamous and ductal epithelium is paramount to the diagnosis (8). The predominant squamous cell carcinoma component which may range from well to poorly differentiated, can present as invasive carcinoma or a carcinoma in situ (1). In the deeper parts, there is an intimate mixture of squamous and adenocarcinoma components which may exist as separate, exclusive zones (9). Adenocarcinomatous component can be tubular, alveolar and/or glandular. The glandular morphology should classically consist of "punched out" spaces with smooth, rather than ragged, edges. Mucin production is typically present but is not a prerequisite for diagnosis in the presence of true gland formation (7).

Acantholytic SCC is an extremely rare variant which is more commonly found on sun-damaged skin of the head and neck and occasionally on the lower lip. Synonyms include adenoid SCC, pseudo-glandular SCC, SCC with gland-like features, angiosarcoma-like SCC and pseudovascular adenoid SCC (10). It was first described by Lever in 1947 as adenocanthoma of eccrine origin until later studies recognized it as SCC of non-eccrine origin. Muller et al. suggested the term "adenoid squamous cell carcinoma" in 1964 to avoid confusion (11). This tumour is characterized by acantholytic or dyscohesive changes in tumour nests at the invasive front, creating a gland-like or adenoid appearance (9). Many of the pseudo-glandular spaces contain acantholytic and dyskeratotic epithelial cells. Mucin stains are always negative in this lesion and evidence of glandular differentiation is absent. In contrast, the duct-like structures in adenosquamous SCC contain intraepithelial or intraluminal sialomucins and the lining cells show ductal differentiation (11). 
Basaloid SCC is a high grade variant with poor prognosis first described by Wain et al. in 1986. Later in 1991, it was included in the WHO's revised classification of tumours of head and neck as a distinct entity (12). The basaloid component is composed of small, hyperchromatic cells with scanty cytoplasm and peripheral palisading arranged into lobular, cord-like, cribiform, tubular and glandular or nest patterns (1). Comedonecrosis is often seen in the central areas of lobules $(3,9)$. Islands of basaloid cells may be surrounded by and contain droplets of PAS-positive hyaline material in a pattern reminiscent of adenoid cystic carcinoma. The hyaline material represents entrapped stroma or excessive basement membrane material $(2,12)$.

The presence of adenoid features necessitates careful exclusion of salivary gland neoplasms. Identifying dysplastic overlying epithelium will help distinguish primary salivary gland neoplasms such as mucoepidermoid carcinoma from SCC and its variants $(4,8)$. In instances where secondary fusion of salivary carcinoma with oral epithelium can simulate a surface origin, the presence of epithelial dysplasia elsewhere in the oral cavity or oropharynx provides circumstantial evidence of a genuine surface origin (2).

On occasions, conventional SCC has been found to extend along salivary ducts where the cancer cells have been found to replace normal ductal cells. Normal ductal luminal cells positively stained with CK7+/CK20 would demonstrate the transition from salivary ducts to SCC whereas squamous cells lining pseudoducts would stain negative $(10,11)$. Conventional SCC entrapping seromucinous glands of the upper respiratory tract may lead to a misdiagnosis of adenosquamous carcinoma. However, the entrapped glands do not show atypical cytological features (6). The present case exhibits notable distance between the neoplastic epithelium and the underlying minor salivary glands which does not favour a primary salivary gland origin (13).

Mucoepidermoid carcinoma is defined by WHO as a malignant glandular epithelial neoplasm characterized by mucous, intermediate and epidermoid cells, with columnar, clear cell and oncocytoid features (14). It frequently affects major salivary glands, most commonly the parotid glands (15). When it occurs in the minor salivary glands, it usually grows beneath the surface epithelium without breaching it or only infiltrating it subsequently (6). Several histological characteristics helpful in distinguishing mucoepidermoid carcinoma from adenosquamous SCC are absence of dysplastic overlying epithelium, lobular arrangement and presence of intermediate cells $(1,6,9,10,16)$. In addition, mucus cells and intraepithelial and ductal sialomucins are readily recognized in mucoepidermoid carcinoma which is not the case here (10).

Adenoid cystic carcinoma (ACC) is a basaloid tumour consisting of epithelial and myoepithelial cells in variable morphologic configurations, including tubular, cribriform and solid patterns. It has a relentless clinical course and usually a fatal outcome (14). Solid variant may resemble basaloid SCC but it should be distinguishable by its characteristic pattern of infiltration, encircling stroma and its preferential perineural spread. There should also be demonstrable ductal structures with a mucinpositive cast in the lumen (2). In solid ACC, comedo necrosis and squamous differentiation is not common while dysplastic surface epithelium is generally absent in ACC (9). Unlike oral SCC, it usually does not have prominent pleomorphism, mitoses or necrosis and tends to metastasize to distant sites rather than cervical lymph nodes (1).

Basal cell adenocarcinoma and salivary duct carcinoma (SDC) merit a mention by virtue of their resemblance to basaloid SCC. Basal cell adenocarcinoma may show squamous differentiation with keratin formation and may merge with or ulcerate the mucosa, raising comparisons with basaloid SCC (2). On the other hand, presence of comedo necrosis in solid areas of SDC may resemble basaloid SCC. The lack of an SCC component and dysplasia in surface epithelium, the associated growth patterns found in SDC and presence of tumour cells with a prominent eosinophilic cytoplasm will help to exclude SDC (9).

In the present case, negative mucin stains with Periodic Acid Schiff and Mucicarmine, coupled with a dysplastic overlying epithelium essentially excluded the possibility of a salivary gland origin or involvement. Absence of true duct formation supports this observation as well as negating adenosquamous carcinoma as a diagnostic possibility. Extensive comedo necrosis noted throughout the lesion resulted in areas with pseudoglandular appearance mimicking that in a basaloid SCC and an acantholytic SCC. However, a lack of basaloid proliferation and convincing acantholytic component in the deeper tumoral tissue components preclude these diagnoses.

It is generally known that in reporting oral malignancy, the absence or presence of vascular, nerve and bone invasions form part of the minimum core dataset. Vascular invasion is a relatively poor predictor of nodal metastasis whereas neural invasion predicts a more aggressive disease. The presence of bone involvement by tumour is important for staging of oral malignancies. In the current case, positive involvement of all three abovementioned structures constitutes important pathological findings which when taken together with the clinical data can be utilized by the surgeon for further post-operative therapeutic assessment and prognostication.

\section{CONCLUSION}

This is a case of well-differentiated squamous cell carcinoma of the right buccal mucosa presenting with 
pseudo-glandular differentiation. It is important to rule out other carcinomas with adenoid features because of the prognostic implications and for appropriate therapeutic decisions.

\section{REFERENCES}

1. Thompson LDR. Squamous cell carcinoma variants of the head and neck. Current Diagnostic Pathology 2003; 9: 384-396

2. Odell EW, Morgan PR. Biopsy pathology of the oral tissues. London: Chapman \& Hall; 1998.

3. Pereira MC, Oliveira DT, Kowalski LP. Histology subtypes of oral squamous cell carcinoma: prognostic relevance. Journal of the Canadian Dental Association 2007; 73 (4): 339-344.

4. Abdelsayed RA, Sangueza OP, Newhouse RF, Singh BS. Adenosquamous carcinoma - a case report with immunohistochemical evaluation. Oral Surg Oral Med Oral Pathol Oral Radiol Endod 1998; 85: 173-7

5. Woolgar JA, Triantafyllou A. Pitfalls and procedures in the histopathological diagnosis of oral and oropharyngeal squamous cell carcinoma and a review of the role of pathology in prognosis. Oral Oncology 2009; 45: 361-385

6. Alos L, Castillo M, Nadal A, Caballero M, Mallofre C, Palacin A, Cardesa A. Adenosquamous carcinoma of the head and neck: criteria for diagnosis in a study of 12 cases. Histopathology 2004; 44: 570-579

7. Masand RP, El-Mofty SK, Ma XJ, Luo YL, Flanagan JJ, Lewis Jr. JS. Adenosquamous carcinoma of the head and neck: relationship to human papilloma virus and review of literature. Head and Neck Pathol 2011; 5: 108-116

8. Scully C, Porter SR, Speight PM, Eveson JW, Gale D. Adenosquamous carcinoma of the mouth: a rare variant of squamous cell carcinoma. Int. J. Oral Maxillofac. Surg 1999; 28: 125-128
9. Heerden WFPV, Zyl AWV. Surgical pathology of oral cancer. Diagnostic histopathology 2009; 15(6)

10. Papadopoulou E, Tosios KI, Nikitakis N, Papadogeorgakis N, Sklavounou-Andrikopoulou A. Acantholytic squamous cell carcinoma of the gingiva: report of a case and review of the literature. Oral Surg Oral Med Oral Pathol Oral Radiol Endod 2010; 109: 67-71

11. Yeoh MS, Kim DD, Ghali GE. Acantholytic squamous cell carcinoma of buccal mucosa: report of a case. J Oral Maxillofac Surg 2012; 70: 17331738

12. Rachel JR, Kumar NS, Jain NK. Basaloid squamous cell carcinoma of retromolar trigone: A case report with review of literature. J Oral Maxillofac Pathol 2011; 15: 192-6

13. Brennand-Roper MJ, Pring M, Hughes CW, Thomas $\mathrm{S}$, Eveson JW. Minor salivary gland squamous cell carcinoma of the lower lip demonstrating striking perineural invasion. Oral Surg Oral Med Oral Pathol Oral Radiol Endod 2010; 110(4): 28-32.

14. Barnes L, Eveson JW, Reichart P, et al. editors. World Health Organization classification of tumours. Pathology and genetics of head and neck tumours. Lyon: IARC Press; 2005.

15. Speight PM, Barrett AM. Diagnostic difficulties in lesions of the minor salivary glands. Diagnostic histopathology 2009; 15(6): 311-317

16. Mokhtari S, Mohsenifar Z, Mokhtari S. Diagnostic histological features of metastatic lymph nodes in adenosquamous carcinoma. Pathology - Research and Practice 2012; 208: 360-362 